\title{
Predicting correlated responses in natural populations: changes in JHE activity in the Bermuda population of the sand cricket, Gryllus firmus
}

\author{
DEREK A. ROFF* $\uparrow \&$ DAPHNE J. FAIRBAIRN $\uparrow$ \\ $\dagger$ Department of Biology, McGill University, 1205 Dr Penfield Ave., Montreal, Quebec, Canada, H3A 1B1 and \\ $\$$ Department of Biology, Concordia University, 1455 de Maisonneuve Blvd., West, Montreal, Quebec, \\ Canada H3G $1 M 8$
}

\begin{abstract}
Quantitative genetic methods have been used to examine selection responses in domesticated organisms but there are few cases of their application to predict changes in natural populations: there are, to our knowledge, no cases in which correlated responses to selection have been predicted. In the present paper we use quantitative genetic parameters estimated from a half-sib experiment to predict the changes expected in juvenile hormone esterase (JHE) activity in the Bermuda population of the wing dimorphic cricket, Gryllus firmus. JHE activity is genetically correlated with wing form in this cricket and hence changes in the proportion of macroptery (volant morph) are predicted to bring about a correlated response in JHE activity. The Bermuda population has a higher proportion of macropterous individuals $(95 \%)$ compared to the stock (35\%, originally from Florida) from which the heritabilities and correlations were estimated. The quantitative genetic analysis makes three predictions which were tested both qualitatively and quantitatively. In all cases the null hypothesis that the observed results correspond to those predicted cannot be rejected. As predicted, in the Bermuda population there is: (i) an increase in the population mean JHE activity; (ii) a leftwards shift in the curve relating the probability of microptery (flightless morph) to JHE activity; and (iii) a decrease in the mean JHE activity within each morph.
\end{abstract}

Keywords: coevolution, correlated response, dimorphism, quantitative genetics, selection.

\section{Introduction}

Quantitative genetic methods have been used extensively in the selection of traits of domesticated animals and plants (Mather \& Jinks, 1982; Falconer, 1989). More recently these methods have been used to study the evolution of traits in natural populations (Roff, 1997; Lynch \& Walsh, 1998). Heritabilities and genetic correlations have been measured in a wide variety of nondomesticated animals (e.g. insects, fish, gastropods, amphibians, birds, mammals) both in the laboratory and in the wild (Mousseau \& Roff, 1987; Roff, 1996; Weigensberg \& Roff, 1996). These studies have shown that there is sufficient additive genetic variation for rapid response to natural selection, as has been observed in several species (Berthold et al., 1992; Grant \& Grant, 1993; Berthold, 1995). In the present paper we use

*Correspondence. E-mail: droff@bio1.lan.mcgill.ca estimates of genetic parameters obtained from a half-sib analysis in the laboratory to predict geographical covariation of two traits (juvenile hormone esterase and proportion of macroptery) among natural populations. Specifically we ask if a quantitative genetic model derived from one population can successfully predict the set of trait values in a geographically distinct population.

Gryllus firmus, the sand cricket, occurs on sandy areas along the eastern coast of North America from Florida as far north as Connecticut (Alexander, 1968; Harrison, 1985), and on the island of Bermuda (Kevan, 1980). This species is wing dimorphic: some individuals possess well developed wings and flight muscles and are capable of flight (macropterous) whereas others have reduced wings, highly reduced or nonexistent flight muscles and are flightless (micropterous: Roff, 1989; Zera et al., 1997). Although there are only two phenotypes, wing dimorphism is polygenic, and can be understood using the threshold model of quantitative genetics (Roff, 1986, 
1997). According to this model the dimorphic trait is determined by a continuously distributed underlying trait, called the liability, and a threshold of sensitivity: if the liability lies below the threshold one morph is produced whereas the alternate morph is produced when the liability is above the threshold (two examples are shown in the top-left panel of Fig. 1). The liability can be viewed as a quantitative trait in the same manner as, say, body size and hence its genetic determination calculated in the same manner (although the statistical protocols have to be modified to take into account that only two phenotypes are observed: see Roff, [1997] for a description of the methodology).

Physiologically the liability could be a single chemical or a complex of chemicals. Circulating chemicals, such as hormones, have been implicated in the production of dimorphic variation in several species, e.g. thyroxin in paedomorphosis (Gould, 1977; pp. 299-302), and testosterone in colour variation in lizards (Hews et al., 1994). Wing dimorphism was hypothesized to be a
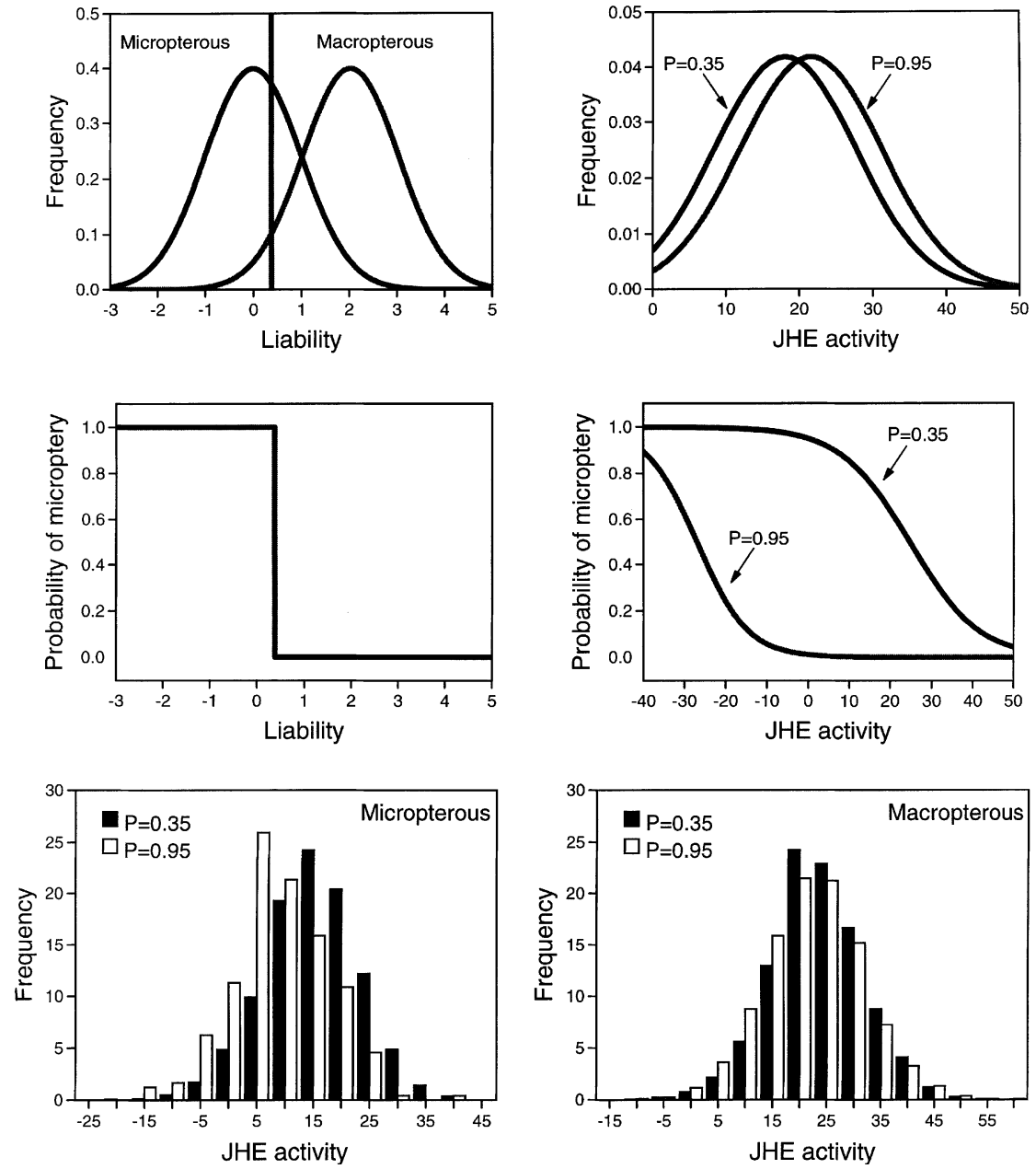

Fig. 1 Three predictions of the quantitative genetic model for the change in JHE activity (nmol of JH converted to JH acid per minute per $\mathrm{mL}$ of haemolymph) accompanying a change in proportion of macroptery in Gryllus firmus. Prediction 1, top row: an increase in proportion of macroptery is generated by a shift in the mean liability, which is accompanied by a smaller shift in the JHE activity. Prediction 2, middle row: The threshold value is not changed and hence the switch point on the liability scale remains unaltered. However, the curve relating the probability of microptery to JHE activity is shifted leftwards. The curves shown were each generated using the following parameter values: $h_{\mathrm{M}}^{2}=0.50 ; h_{\mathrm{JHE}}^{2}=0.21 ; r_{\mathrm{G}}=0.28 ; r_{\mathrm{P}}=0.58 ; \mu_{\mathrm{JHE}}$ (at proportion of macroptery, $P=0.35)=18 ; \sigma_{\mathrm{JHE}}^{2}=91$. In each case 5000 individual values were obtained using the eqns (4) and (5) presented in the text and a logistic curve fitted to the data so obtained. Prediction 3, bottom row: the mean JHE activity within morphs will decline as the frequency of macroptery is increased. The histograms shown were constructed using the data produced as described in Prediction 2. Means obtained for this simulation were as follows (expected values in parentheses): micropterous, $P=0.35$, $\overline{J H E}=15.1$ (14.8) or $P=0.95, \overline{J H E}=8.8(10.1)$; macropterous, $P=0.35, \overline{J H E}=23.6(23.9)$ or $P=0.95, \overline{J H E}=22.3(22.1)$. 
consequence of variation in the level of juvenile hormone (Southwood, 1961; Wigglesworth, 1961). In the cricket Gryllus rubens the level of $\mathrm{JH}$ production between presumptive wing morphs does not differ but the activity of juvenile hormone esterase (JHE; the principal enzyme involved in degradation of $\mathrm{JH}$ in the haemolymph) is significantly different, presumptive macropterous nymphs having a higher activity of JHE (and thus a lower concentration of $\mathrm{JH}$ ) than presumptive micropterous nymphs in the final instar (Zera et al., 1989; Zera \& Tobe, 1990; Zera \& Holtmeier, 1992). Similar variation in JHE level relative to wing morphology has been observed in G. firmus (Fairbairn \& Yadlowski, 1997; Roff et al., 1997). These observations are consistent with the hypothesis that the JHE activity plays an important role in the determination of adult wing morphology, and further suggest that JHE might be a significant component of the liability. A genetic analysis of wing morphology and JHE in G. firmus showed that JHE cannot by itself by considered the liability (Roff et al., 1997). Because JHE does not show a one-to-one mapping with the liability, JHE is best analysed as a character that is genetically correlated with the liability. A significant genetic correlation between wing morph and JHE has been demonstrated in G. firmus from both pedigree analysis (Roff et al., 1997) and directional selection on the proportion of macroptery (Fairbairn \& Yadlowski, 1997).

The above analyses on G. firmus were made using a stock derived from a population in northern Florida. Field data indicate that the proportion of macroptery is higher in the Bermuda population than elsewhere (Kevan, 1980). This population is undoubtedly a result of colonization from the mainland, where populations surveyed show a lower proportion of macroptery (Veazey et al., 1976; Kevan, 1980; Roff, unpubl. data). Thus selection in Bermuda has probably favoured an increase in proportion of macroptery. Alternatively, there may be no selection on macroptery in the Bermuda population and the present high proportion is a consequence of the initial colonizers being macropterous. In this case the initial colonizing event represents a single episode of directional selection.

An important test of the utility of the quantitative genetic analysis of the genetic architecture of wing morphology and JHE in describing the evolution of the two traits is whether it can accurately predict levels of JHE in the Bermuda population based on the observed difference in proportion of macroptery. The genetic correlation between JHE and wing morph and the underlying physiological model for wing morph determination lead to the qualitative prediction that evolutionary changes in proportion of macroptery will be accompanied by (and, in fact, functionally determined by) changes in JHE activity. We test this hypothesis quantitatively using the threshold model of wing morph determination and estimates of genetic parameters obtained from the half-sib analysis of G. firmus from the Florida population.

\section{Three predictions}

Using quantitative genetic theory we develop three predicted differences between the Florida and Bermuda stocks. These predictions are illustrated in Fig. 1. It is important to note that in the following analyses we are not assuming that the Bermuda population was derived from the Florida population: the equations predict the difference between the two populations using the Florida population as the reference population (this is mathematically equivalent to deriving the predictions for the two populations from some common ancestor and then simply comparing the two descendent populations).

Prediction 1: an increase in proportion of macroptery will be associated with an increase in the mean JHE activity.

The mean JHE activity associated with a given change in macroptery can be computed using the standard formulae for direct and correlated responses (Falconer, 1989):

$R_{\mathrm{M}}=i h_{\mathrm{M}}^{2} \sigma_{\mathrm{M}}$

$C R_{\mathrm{JHE}}=i r_{\mathrm{G}} h_{\mathrm{M}} h_{\mathrm{JHE}} \sigma_{\mathrm{JHE}}$,

where $R_{\mathrm{M}}$ is the direct response of selection on the proportion of macroptery $\left(R_{\mathrm{M}}\right.$ is measured on the underlying scale, the liability), $i$ is the intensity of selection, $h_{\mathrm{M}}^{2}$ is the heritability of wing morph (strictly, this is the heritability of the continuously distributed underlying trait, the liability), $\sigma_{\mathrm{M}}^{2}$ is the phenotypic variance, which by definition equals 1 (Roff, 1994), $C R_{\mathrm{JHE}}$ is the correlated response of JHE activity, $r_{\mathrm{G}}$ is the additive genetic correlation between the liability of wing morph and JHE activity, $h_{\mathrm{JHE}}$ is the square root of the heritability of JHE activity and $\sigma_{\mathrm{JHE}}^{2}$ is the phenotypic variance of JHE activity. Rearranging the above to eliminate the intensity of selection gives

$C R_{\mathrm{JHE}}=r_{\mathrm{G}} R_{\mathrm{M}}\left(h_{\mathrm{JHE}} \sigma_{\mathrm{JHE}} / h_{\mathrm{M}} \sigma_{\mathrm{M}}\right)$.

The predicted mean JHE activity equals the initial mean plus $C R_{\mathrm{JHE}}$. An increase in the proportion of macroptery requires a positive $R_{\mathrm{M}}$ and thus, because all other terms in the above equation are positive, an increase in proportion of macroptery will be 
accompanied by an increase in the mean JHE activity (see top panels of Fig. 1). To determine the magnitude of the change expected, we used parameter values obtained from a half-sib experiment using the Florida stock (Roff et al., 1997) and a change in proportion of macroptery from $35 \%$ to $95 \%$, which matches approximately the variation between the Florida and Bermuda stocks (see Results).

For the initial population (35\%) the mean liability is arbitrarily set at 0 and the threshold, $T$, set at the value required to produce $35 \%$ macropterous individuals $\left(0.35=\int_{T}^{\infty} \phi(x) \mathrm{d} x\right.$, where $\phi(x)$ is the standard normal deviate, from which $T=0.383$ ). Because the initial mean liability is zero (by definition), $R_{\mathrm{M}}$ is equal to the mean liability at the new proportion of macroptery. The mean liability, $\mu_{\mathrm{M}}$, required to produce $95 \%$ macroptery, is obtained by solving the equation $0.95=\int_{0.383}^{\infty} \phi\left(\begin{array}{ll}x & \mu_{\mathrm{M}}\end{array}\right) \mathrm{d} x$, from which we obtain $R_{\mathrm{M}}=2.033$. Substitution in eqn 3 gives a correlated change in JHE activity of $3.51 \mathrm{nmol}$ of JH converted to $\mathrm{JH}$ acid per minute per $\mathrm{mL}$ of haemolymph (for simplicity we shall hereafter designate the units as nmols/min).

Prediction 2: an increase in proportion of macroptery will be associated with a shift to the left in the curve relating the probability of being micropterous to JHE activity.

Because of the threshold, the curve relating the probability of being micropterous to the liability is a 0,1 step function, independent of the distribution of the liability (middle-left panel of Fig. 1). However, for any given JHE activity both morphs are possible, with the probability of microptery decreasing as JHE activity increases (middle-right panel of Fig. 1). Furthermore, this curve does depend upon the distribution of the liability.

The values of liability and JHE activity for individuals can be specified by the equations (Roff \& Bradford, 1998):

$$
\begin{aligned}
X_{i}= & \mu_{\mathrm{M}}+a_{x, i} \sqrt{\frac{1}{2} h_{\mathrm{M}}^{2}}+b_{x, i} \sqrt{1 \frac{1}{2} h_{\mathrm{M}}^{2}} \\
Y_{i}^{*}= & r_{\mathrm{G}} a_{x, i} \sqrt{\frac{1}{2} h_{\mathrm{JHE}}^{2}}+r_{\mathrm{E}} b_{x, i} \sqrt{1 \frac{1}{2} h_{\mathrm{JHE}}^{2}} \\
& +a_{y, i} \sqrt{\frac{\left(1 \frac{\left.r_{\mathrm{G}}^{2}\right) h_{\mathrm{JHE}}^{2}}{2}\right.}{2}+b_{y, i} \sqrt{\left.1 \frac{1}{2} h_{\mathrm{JHE}}^{2}\right)\left(1 \quad r_{\mathrm{E}}^{2}\right)}} \\
Y_{i}= & \mu_{\mathrm{JHE}}+\sigma_{\mathrm{JHE}} Y_{i}^{*},
\end{aligned}
$$

where: $X_{i}$ is the liability for the $i$ th individual; $Y_{i}$ is the JHE activity for the $i$ th individual; $a_{x, i}, a_{y, i}, b_{x, i}$ and $b_{y, i}$ are random standard normal values, $\mathrm{N}(0,1) ; \mu_{\mathrm{M}}$ is the mean liability; $\mu_{\mathrm{JHE}}$ is the mean JHE activity; and $r_{\mathrm{E}}$ is the environmental correlation, given by

$r_{\mathrm{E}}=\frac{r_{\mathrm{P}} \frac{1}{2} r_{\mathrm{G}} \sqrt{h_{\mathrm{M}}^{2} h_{\mathrm{JHE}}^{2}}}{\sqrt{\left.\left.1 \frac{1}{2} h_{\mathrm{M}}^{2}\right) 1 \frac{1}{2} h_{\mathrm{JHE}}^{2}\right)}}$,

where $r_{\mathrm{P}}$ is the phenotypic correlation.

For a given JHE activity the probability of an individual being micropterous is equal to the probability that the liability is less than the threshold $\left(X_{i}<T\right)$. This probability is decreased with an increase in macroptery by virtue of the increased value of the mean liability $\mu_{\mathrm{M}}$. Thus the probability curve should be shifted to the left of the original curve. We numerically estimated the curves relating JHE activity and probability of microptery by (i) generating 5000 individuals per population (macroptery $=0.35$ and 0.95 ) according to the above algorithm and (ii) fitting a logistic model to these data (Fairbairn \& Yadlowski, 1997). The logistic model was fitted using maximum likelihood, with the parameter values tested using the estimated $t$-values. All coefficients in the fitted model were highly significant $(P<0.00005)$. As predicted, the curve for the population consisting of $95 \%$ macropterous individuals is shifted to the left, the shift being very substantial (Fig. 1): thus, for example, when JHE activity $=20$ $\mathrm{nmols} / \mathrm{min}$ and proportion of macroptery $=0.35$, the probability of being micropterous is 0.64 , whereas when the proportion of macroptery $=0.95$ the probability is only 0.0004 . There is a slight change in the slopes of the two curves, the regression equation being

$y=0.5836+0.12 x+4.29 P o p+0.04(x)(P o p)$,

where $x$ is JHE activity measured as the residual from the overall mean, Pop is a dummy variable specifying the population ( $=0$ for $35 \%$ macroptery, $=1$ for $95 \%$ macroptery) and the probability of being micropterous is given by

$P($ micropterous $)=\frac{1}{1+e^{y}}$.

Prediction 3: whereas an increase in the proportion of macroptery will be associated with an increase in the mean JHE activity (Prediction 1), the mean JHE activity within morphs will be decreased.

Consider the above model; if $X_{i}$ exceeds $T$ then the individual is macropterous. Thus to compute the mean JHE activity within the macropterous morph we must compute the expected value of $Y_{i}$ for the case $X_{i}>T$. The components of $Y_{i}$ that have coefficients $a_{y, i}$ and $b_{y, i}$ 
have expectations of zero and do not influence the value of $X_{i}$ and hence can be dropped. The expected JHE activity among macropterous individuals is given by

$$
\left(\frac{\int_{\infty}^{\infty} \int_{f(a)}^{\infty}\left(r_{\mathrm{G}} a \sqrt{0.5 h_{\mathrm{JHE}}^{2}}+r_{\mathrm{E}} b \sqrt{10.5 h_{\mathrm{JHE}}^{2}}\right) \phi(b) \phi(a) \mathrm{d} b \mathrm{~d} a}{\int_{\infty}^{\infty} \int_{f(a)}^{\infty} \phi(b) \phi(a) \mathrm{d} b \mathrm{~d} a}\right)
$$

where $f(a)=\frac{T \mu_{\mathrm{M}} a \sqrt{0.5 h_{\mathrm{M}}^{2}}}{\sqrt{10.5 h_{M}^{2}}}$ and $\phi(\cdot)$ is the standard normal deviate. The mean value of JHE activity within the micropterous morph is determined by setting the limits of integration of the second integral from $-\infty$ to $f(a)$. Using the estimated parameter values the mean JHE activity within the macropterous morph changes from $23.8 \mathrm{nmols} / \mathrm{min}$ when the proportion of macroptery is 0.35 , to $22.1 \mathrm{nmols} / \mathrm{min}$ when the proportion of macroptery is 0.95 . Likewise the mean JHE activity within the micropterous morph changes from 14.8 $\mathrm{nmols} / \mathrm{min}$ when the proportion of macroptery is 0.35 , to $10.1 \mathrm{nmols} / \mathrm{min}$ when the proportion of macroptery is 0.95 . Thus there is a greater change in the mean JHE activity within the micropterous morph (Fig. 1).

Unlike the previous two predictions in which the direction of change does not depend on the parameter estimates, in the present case the directions of the two responses are contingent on parameter values. To examine the robustness of the present predictions we selected 1000 combinations of parameter values assuming normal distributions with the means and standard errors from the half-sib experiment: $h_{\mathrm{M}}^{2}=0.50, \mathrm{SE}=0.12$; $h_{\mathrm{JHE}}^{2}=0.21, \quad \mathrm{SE}=0.10 ; \quad r_{\mathrm{P}}=0.58, \quad \mathrm{SE}=0.03 ; \quad r_{\mathrm{G}}=$ $0.28, \mathrm{SE}=0.44$ (all sire estimates). The standard error for the genetic correlation is very large but the significant correlated response of JHE activity with selection on proportion of macroptery observed by Fairbairn \& Yadlowski (1997) demonstrated that there is a positive genetic correlation between JHE activity and the liability. Because of the latter observation we assumed that $r_{\mathrm{G}}$ was positive, setting values less than 0.01 to 0.01 . The mean change in JHE activity within morphs was $4.88 \mathrm{nmols} / \mathrm{min}$ (median $=6.09 \mathrm{nmols} / \mathrm{min}$ ) for the micropterous morph and $0.89 \mathrm{nmols} / \mathrm{min}$ (median $=1.99 \mathrm{nmols} / \mathrm{min})$ for the macropterous morph. In $84 \%$ of the combinations the mean JHE activity within the micropterous morph declined, whereas in the macropterous morph a decline occurred in $64 \%$ of cases. We conclude that a decline in mean JHE activity is more likely in the micropterous morph than in the macropterous morph.

\section{Materials and methods}

To test the above three predictions we compared the JHE activity of female G. firmus from two populations differing in proportion of macroptery, one from Bermuda and one from Florida. The Bermuda stock was derived from 95 (31 females, 36 males, 28 nymphs) G. firmus collected in August 1997. This stock was maintained at $30^{\circ} \mathrm{C}$ for approximately three generations before eggs were collected for the present experiment. The Florida stock was initiated from $\approx 20$ females and 20 males collected from northern Florida in 1981.

For the present experiment crickets from each stock were raised under two environmental conditions: (i) $28^{\circ} \mathrm{C}$ and a photoperiod of $12 \mathrm{~L}: 12 \mathrm{D}$, which corresponds approximately to early or late summer conditions; (ii) $30^{\circ} \mathrm{C}$ and $14 \mathrm{~L}: 10 \mathrm{D}$, which corresponds to midsummer conditions (we hereafter refer to these two environments simply by their temperature). For each combination of population and environment 10 cages (4 L buckets) of 40 nymphs per cage were established and reared according to the husbandry methods detailed in Roff (1986). Wing morph is correlated with JHE activity in the final nymphal instar, although it is only in the adult that wing morphology can be visually determined (Fairbairn \& Yadlowski, 1997). Previous studies of G. firmus and G. rubens have shown that JHE activity reaches a peak in the middle of the final instar, which corresponds to day 5 at $28^{\circ} \mathrm{C}$ (Zera \& Tiebel, 1989; Fairbairn \& Yadlowski, 1997): because the peak may vary according to temperature, photoperiod or population we assayed JHE activity on days 3,5 and 7 of the final instar. JHE activity was assayed following the protocol described in Roff et al. (1997). Males do not differ from females in mean JHE activity or the relationship between JHE activity and wing morph (Fairbairn \& Yadlowski, 1997). However, to eliminate any possible confounding influence of sex we used only females for these analyses. A minimum of 21 females per population per day was assayed $(21<n<54)$, with individuals being drawn in approximately equal numbers from each cage. After being bled to obtain a haemolymph sample, nymphs were raised individually until final eclosion, at which time wing morph was scored. Haemolymph removal itself appears to increase the frequency of the micropterous morph (Fairbairn \& Yadlowski, 1997), and so the frequency of macroptery was obtained both from the group of bled individuals and from another experiment in which individuals were neither bled nor raised apart for the last few days of their development, but otherwise were treated in the same manner (obtained from S. Mostowy, unpubl. data). 


\section{Results}

\section{Is the proportion of macroptery higher in the Bermuda stock?}

Each cage was treated as an individual datum by calculating for each the proportion of macroptery, which was then arcsine-square root transformed. As expected, the Bermuda population produced a higher frequency of macropterous females $\left(85 \%\right.$ at $28^{\circ} \mathrm{C} ; 96 \%$ at $\left.30^{\circ} \mathrm{C}\right)$ than the Florida stock $\left(12 \%\right.$ at $28^{\circ} \mathrm{C} ; 46 \%$ at $30^{\circ} \mathrm{C}$ ). A two-way ANOva indicated no environmentby-population interaction $\left(F_{1,36}=2.87 ; P=0.099\right)$, but highly significant effects of environment $\left(F_{1,36}=14.40\right.$, $P=0.001)$ and population $\left(F_{1,36}=86.52, P<0.0005\right)$. Results from the unbled animals gave similar results (Bermuda: $99 \%$ at $28^{\circ} \mathrm{C} ; 93 \%$ at $30^{\circ} \mathrm{C}$; Florida: $34 \%$ at $28^{\circ} \mathrm{C} ; 40 \%$ at $30^{\circ} \mathrm{C}$; S. Mostowy, unpubl. data). Bleeding the nymphs for the haemolymph has been shown to induce microptery in individuals that would otherwise moult into macropters when reared at $28^{\circ} \mathrm{C}$ (Fairbairn \& Yadlowski, 1997). We observed the same effect at $28^{\circ} \mathrm{C}$ (proportion of macroptery: 0.85 vs. 0.99 , and 0.12 vs. 0.34 ) but not at $30^{\circ} \mathrm{C}$ (proportion of macroptery: 0.96 vs. 0.93 and 0.46 vs. 0.40 ).

\section{Prediction 1: an increase in proportion of macroptery will be accompanied by an increase in the mean JHE activity}

Because JHE activity varied with age we analysed the data using residuals from the overall mean for each age and also using the standardized residuals, obtained by dividing the residuals by the standard deviation at each age (Fairbairn \& Yadlowski, 1997). The results were the same for both methods and we report here the analyses based on the residuals. Analyses were first carried out with the data nested within cages: as there was no effect of cage the data were combined.

In accordance with the above prediction, the mean JHE activity at all ages was greater in the Bermuda than the Florida stock (Fig. 2). A two-way ANova on the residuals indicated no significant interaction between environment and population $\left(F_{1,401}=2.05, P=0.153\right)$, nor an effect of environment alone $\left(F_{1,401}<0.0005\right.$, $P=0.996)$. There was, however, a highly significant effect of population $\left(F_{1,401}=95.77, P<0.0005\right)$.

The mean separation between the two populations was $7.8 \mathrm{nmols} / \mathrm{min}$, which is significantly larger $(95 \%$ confidence interval $=6.3-9.3 \mathrm{nmols} / \mathrm{min}$ ) than that predicted by the quantitative genetic model using the estimated parameter values $(3.5 \mathrm{nmols} / \mathrm{min})$. The preceding test does not take into account variation in the predicted value stemming from uncertainty in the parameter estimates. To estimate the $95 \%$ confidence region for the prediction we used the 1000 combinations of parameter values described previously: these gave an interval of $0.12-15.6 \mathrm{nmols} / \mathrm{min}$, which includes the observed value. The relatively large interval is primarily
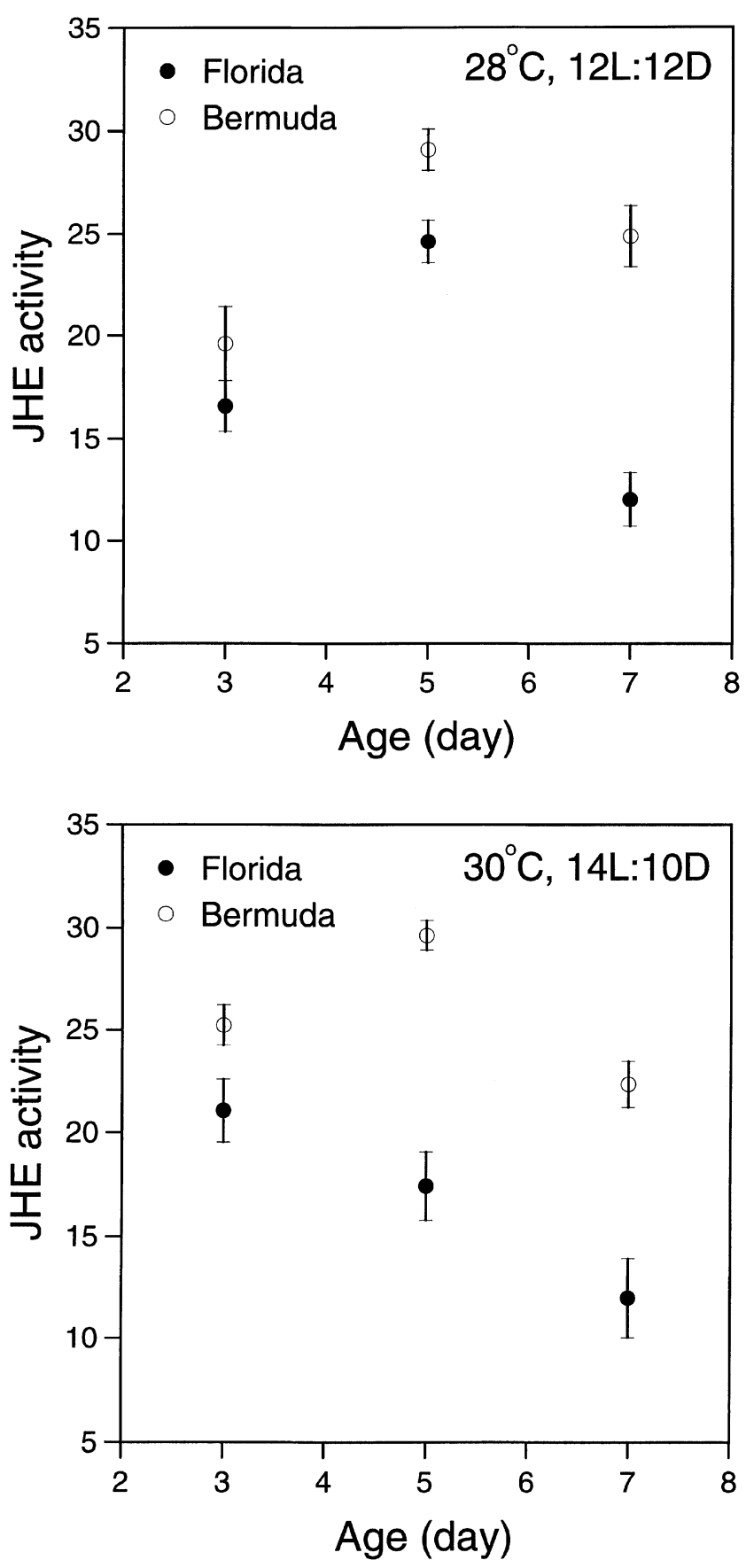

Fig. 2 JHE activity (nmol of JH converted to JH acid per min per $\mathrm{mL}$ of haemolymph, bars show $\pm 1 \mathrm{SE}$ ) as a function of age in the last instar, environmental conditions $\left(28^{\circ} \mathrm{C}\right.$ and 12L:12D vs. $30^{\circ} \mathrm{C}$ and 14L:10D), and population of Gryllus firmus.

(C) The Genetical Society of Great Britain, Heredity, 83, 440-450. 
a consequence of the large confidence region for the genetic correlation (see earlier). Because of the large confidence interval the quantitative prediction of the model cannot be tested satisfactorily.

\section{Prediction 2: the curve relating the probability of being micropterous to JHE activity will be shifted to the left}

Because of the apparent effect of bleeding on proportion of macroptery in the $28^{\circ} \mathrm{C}$ environment, we analysed the two environments separately. We first analysed the data independently of the prediction and then compared the logistic regressions from the quantitative genetic model with those obtained from fitting to the data.

The overall fit of the logistic model was determined using the $\log$ likelihood ratio $D=2(\operatorname{LL}(k)-\operatorname{LL}(0))$, where $\operatorname{LL}(k)$ is the maximum log likelihood with $k$ fitted parameters $(=4)$ and $\operatorname{LL}(0)$ is the log likelihood value for the constants only model. The statistic $D$ is distributed as a $\chi^{2}$ with $k-1$ degrees of freedom (Dobson, 1983). A significant improvement in fit of the model is shown by a significant $\chi^{2}$ value. The significance of the coefficients was evaluated by computing the $t$-values based on the maximum likelihood estimates and estimated standard errors.

In both environments the logistic model gave a highly significant fit to the data $\left(30^{\circ} \mathrm{C}, D=160\right.$, d.f. $=3$, $P<0.00005 ; 28^{\circ} \mathrm{C}, D=166$, d.f. $=3, P<0.00005$ ), but in neither case was the interaction term significant $(P>0.2$, in both environments). Both residual JHE and population were highly significant $\left(30^{\circ} \mathrm{C}\right.$, $P<0.00005$ for both coefficients; $28^{\circ} \mathrm{C}, P<0.00005$ for population and $P=0.0003$ for JHE residual). As predicted, the curves for the Bermuda population were shifted to the left of the Florida curves (Fig. 3).

The null hypothesis of no difference between the logistic regression obtained from the data and that predicted by the quantitative genetic model can be rejected if $\operatorname{LL}(k)-L_{L}$ Model $>\chi^{2} / 2$, where $\operatorname{LL}(k)$ is as defined above, $\mathrm{LL}_{\text {Model }}$ is the $\log$ likelihood obtained using the quantitative genetic model, and $\chi^{2}$ is the upper 0.05 point value of the chi-squared distribution with $k$ d.f. (Cox \& Hinkley, 1974). The number of parameters in the present model is four, for which the critical $\chi^{2}$ value is 9.488 (the following conclusions remain the same if we consider only the three-parameter additive model). Thus values of $L L(4)-L_{\text {Model }}$ that are less than 4.744 are not significant. For the $30^{\circ} \mathrm{C}$ environment, $\mathrm{LL}(4)-\mathrm{LL}_{\text {Model }}=2.803$, indicating that the difference between the observed best fit and the fit of the quantitative genetic model was not significant, as is evident from the predicted and observed curves (Fig. 3).
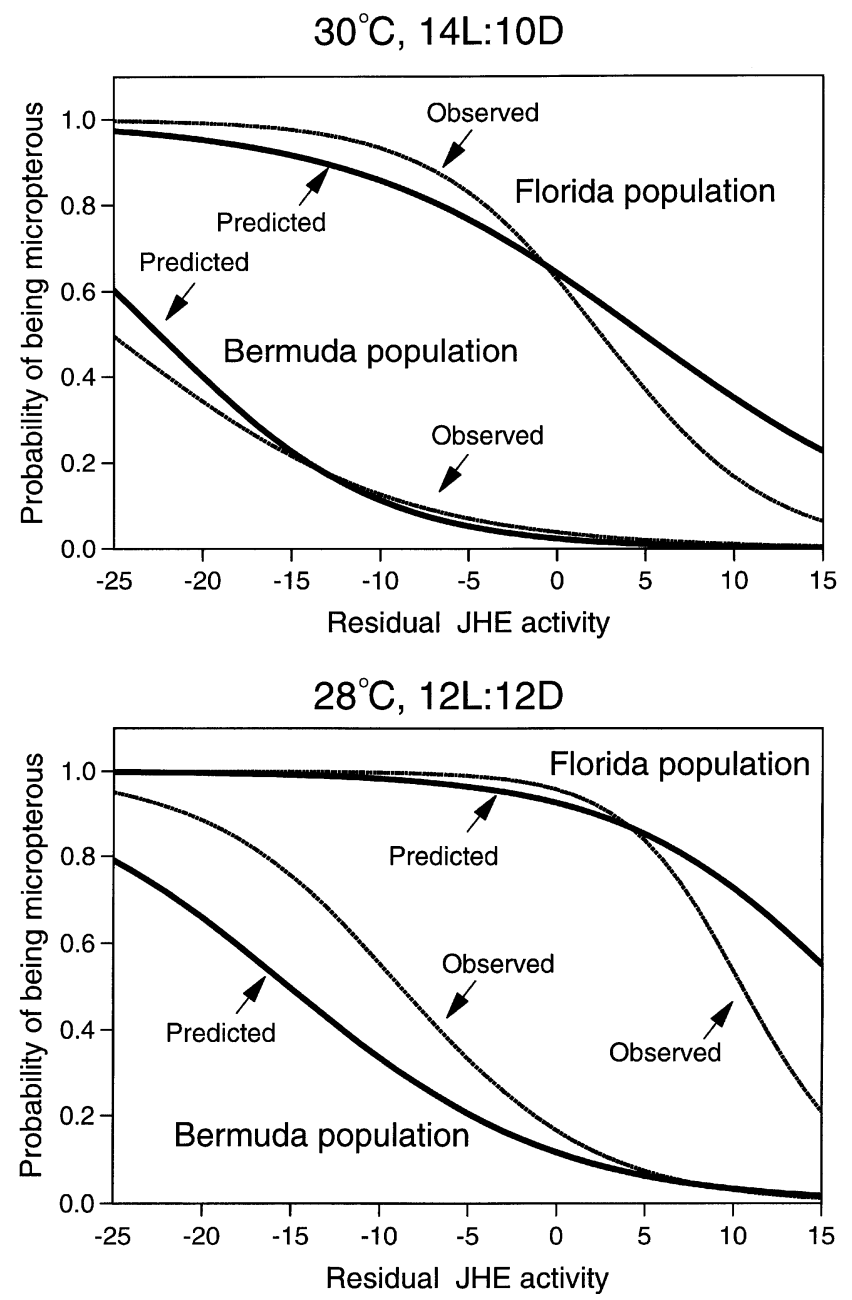

Fig. 3 Logistic curves relating the probability of being micropterous to the residual JHE activity in Gryllus firmus. Predicted, curves predicted from the quantitative genetic model. Observed, curves obtained by logistic regression using the observed JHE residuals.

For the $28^{\circ} \mathrm{C}$ environment it was necessary to take into account the effect of bleeding. To do this we adjusted the phenotypic mean liability in the numerical model such that the proportion of macropterous individuals matched that observed. This adjustment meant that presumptive macropterous individuals lying close to the threshold in the absence of bleeding moulted into micropterous adults with bleeding. The adjustment did not affect the predicted change in JHE activity. The predicted curve for Bermuda tended to overestimate the probability of becoming micropterous at low residual JHE values, whereas the predicted curve for the Florida population underestimated the probability for high residual JHE values (Fig. 3). Nevertheless, the value of $\operatorname{LL}(4)$ - $\mathrm{LL}_{\text {Model }}(=3.658)$ was nonsignificant, indicating a statistically satisfactory fit. 
Prediction 3: whereas the mean JHE activity in the population is increased (Prediction 1), the mean JHE activity within morphs is decreased

Within each environment there are four observed and predicted JHE residuals (the predicted values for the $28^{\circ} \mathrm{C}$ were obtained using the bleeding correction as described above). To compare observed and predicted values we computed the regression of observed on predicted value (Fig. 4). In both environments the model correctly predicted that macropterous individuals would have higher levels of JHE activity than micro-
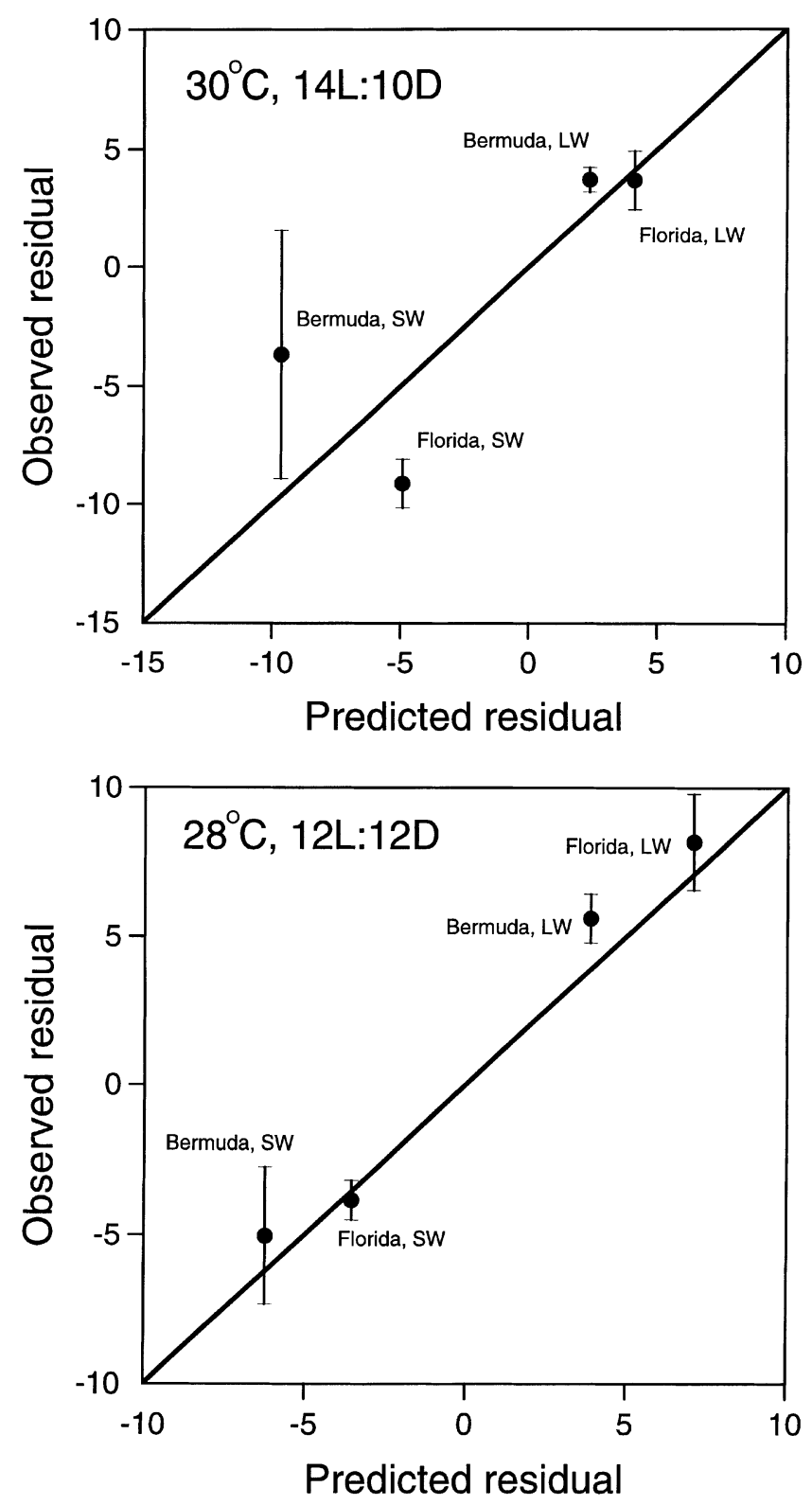

Fig. 4 Observed and predicted JHE mean residuals ( $\pm 1 \mathrm{SE}$ ) within morphs of Gryllus firmus. pterous individuals (Fig. 4). For the $30^{\circ} \mathrm{C}$ environment the correlation between observed and predicted was not significant $(r=0.78, P=0.22)$, and contrary to prediction, within each morph the Bermuda crickets had higher JHE levels than the Florida crickets, although in neither case was the difference statistically significant. The correlation between predicted and observed values was highly significant in the $28^{\circ} \mathrm{C}$ environment $(r=0.99, P=0.007)$, and the values fell close to the 1:1 line (Fig. 4). Further, the direction of difference in JHE activity within morphs between populations was as predicted.

\section{Reversing the procedure to estimate parameter combinations}

We can reverse the procedure previously followed and ask the question: "what combinations of parameter values will produce predicted values that lie within the $95 \%$ confidence regions of the observed data?' There are six such confidence regions: one for prediction 1, one for prediction 2 (we shall ignore the data set requiring adjustment for the effect of bleeding), and four for prediction 3 (again ignoring the case requiring adjustment for the effect of bleeding). We limited the parameter combinations to those defined by the confidence limits obtained from the half-sib experiment.

From the 1000 combinations of parameter values previously described, 13 gave predictions falling within the $95 \%$ confidence limits of the data (Table 1). The genetic correlation ranges from 0.43 to 0.97 , reflecting the large standard error of the estimate from the half-sib

Table 1 Combinations of the heritability of wing morph $\left(h_{\mathrm{M}}^{2}\right)$, the heritability of JHE $\left(h_{\mathrm{JHE}}^{2}\right)$ and the genetic correlation between them $\left(r_{\mathrm{G}}\right)$ that give predictions that fall within the $95 \%$ confidence intervals of all observations made on Gryllus firmus

\begin{tabular}{llll}
\hline$r_{\mathrm{G}}$ & $h_{\mathrm{M}}^{2}$ & $h_{\mathrm{JHE}}^{2}$ & $r_{\mathrm{G}} \frac{h_{\mathrm{HE}}}{h_{\mathrm{M}}}$ \\
\hline 0.43 & 0.38 & 0.31 & 0.39 \\
0.45 & 0.29 & 0.23 & 0.4 \\
0.57 & 0.44 & 0.25 & 0.43 \\
0.61 & 0.44 & 0.21 & 0.42 \\
0.62 & 0.53 & 0.21 & 0.39 \\
0.67 & 0.57 & 0.21 & 0.41 \\
0.67 & 0.43 & 0.15 & 0.39 \\
0.68 & 0.56 & 0.22 & 0.42 \\
0.74 & 0.53 & 0.14 & 0.38 \\
0.75 & 0.62 & 0.16 & 0.38 \\
0.83 & 0.54 & 0.14 & 0.42 \\
0.83 & 0.51 & 0.11 & 0.39 \\
0.97 & 0.47 & 0.08 & 0.41 \\
Means: 0.68 & 0.49 & 0.19 & - \\
\hline
\end{tabular}


experiment, but $46 \%$ fall within the range $0.5-0.6$. The heritabilities show less variation with $85 \%$ of $h_{\mathrm{M}}^{2}$ lying in the range $0.4-0.6$ and $69 \%$ of $h_{\mathrm{JHE}}^{2}$ lying in the range $0.15-0.25$ (Table 1). The appropriate combinations are not free to vary because the correlated response is proportional to $r_{\mathrm{G}}\left(h_{\mathrm{JHE}} / h_{\mathrm{M}}\right)$ (see eqn 3 ): this is shown by all 13 combinations giving values of the foregoing relationship between 0.38 and 0.42 . It is thus not strictly correct to obtain overall estimates by averaging the 13 values, but with this caveat it is instructive to note that the averaged heritability estimates are both close to their estimated values (Table 1). The averaged value for the genetic correlation is 0.68 , which is larger than the estimate from the half-sib experiment (0.28).

\section{Discussion}

The quantitative genetic model of the inheritance of liability and JHE activity in G. firmus made three predictions, each prediction comprising a qualitative and quantitative component. Prediction 1 was that the JHE activity of the Bermuda population would be higher than that of the Florida population (Fig. 1). This prediction depends only upon the heritabilities and genetic correlation being positive. As predicted, the Bermuda population did have a higher JHE activity (Fig. 2). Using the estimated parameter values the separation between the two populations was predicted by the quantitative genetic model to be $3.5 \mathrm{nmols} / \mathrm{min}$ whereas the observed value was $7.8 \mathrm{nmols} / \mathrm{min}$. Taking into account variation in the original parameter estimates and the observed variation, the null hypothesis of no difference between predicted and observed could not be rejected, although the observed difference did suggest that the estimate of the genetic correlation was probably low.

Prediction 2 was that the curve relating the probability of being micropterous to JHE activity would be shifted leftwards in the Bermuda population (Fig. 1). This prediction was verified (Fig. 3). Additionally, the quantitative genetic predictions were not significantly different from the maximum likelihood fit to the observed data. Prediction 3 did depend upon parameter values and an examination of 1000 parameter combinations showed that in $84 \%$ of cases the mean JHE activity within the micropterous morph was predicted to decline whereas a decline in the macropterous morph was predicted in $64 \%$ of the combinations. There was a very good fit between prediction and observation in one environment $\left(28^{\circ} \mathrm{C}\right.$, Fig. 4) but in the other environment the results were inconclusive. Although we cannot explain the environment effect, it may be significant that our predictive parameters were based on data collected at $28^{\circ} \mathrm{C}$.

We estimated, using the 1000 randomly assigned combinations of parameters, which combinations of parameter values produced predictions that matched all observations. Thirteen combinations satisfied all requirements (Table 1). The heritabilities were very similar to those estimated from the half-sib experiment but the required genetic correlation was larger than previously estimated. There are two lines of evidence that support the hypothesis that a genetic correlation of 0.28 is low. First, the dam estimate in the half-sib experiment was $0.90(\mathrm{SE}=0.20)$. This could be a result of nonadditive or maternal effects, but given the large standard error of the sire estimate (0.44), the hypothesis of no such effects cannot be rejected. The confidence region from the dam estimate $(0.5-1.0)$ includes 11 of the 13 'acceptable' combinations. The second line of evidence suggesting a relatively large genetic correlation is the highly significant correlated response of JHE activity to selection on proportion of macroptery (Fairbairn \& Yadlowski, 1997).

In their analysis, Fairbairn \& Yadlowski (1997) concluded from the logistic regression analysis that the changes in JHE activity were most probably brought about by a change in both the mean liability and threshold. Although the present results cannot say that this did not occur in the selected lines, under the correlated trait model it is not necessary to hypothesize that a change in the intercept and slope of the logistic regression is caused by a shift in the threshold. A change in both location and slope is predicted by the quantitative model with JHE as a trait correlated with the liability (Fig. 1). We did not observe a significant change between the Florida and Bermuda populations but Fairbairn \& Yadlowski (1997) did observe such a shift in the selected populations.

Provided the heritabilities of the liability and JHE activity are positive and the genetic correlation between them is positive, we expect an increase in mean JHE activity with an increase in proportion of macroptery (eqn 2). However, changes within the morphs do depend upon the particular combination of parameter values (eqn 7). Examples of the possible changes are shown in Table 2. Under the parameter values estimated from the half-sib experiment the mean JHE activity within each morph is lower at $95 \%$ macroptery than at $35 \%$ macroptery, the overall increase in JHE activity being primarily a consequence of the changing proportion of macropterous individuals. However, if the genetic correlation is large $\left(r_{\mathrm{G}}=0.90\right)$ the mean JHE activity within morphs increases in both cases, whereas if both the genetic and phenotypic correlations are large $\left(r_{\mathrm{G}}=0.90, r_{\mathrm{P}}=0.95\right)$ the mean JHE activity decreases within the micropterous morph but increases within the macropterous morph. Note that the mean JHE activity of the population does not depend upon the value of the phenotypic correlation, whereas the mean within 
Table 2 Effect of the genetic and phenotypic correlations on the predicted mean values of JHE activity (nmol of JH converted to $\mathrm{JH}$ acid per minute per $\mathrm{mL}$ of haemolymph) in Gryllus firmus when the proportion of macroptery is increased from 0.35 to 0.95

\begin{tabular}{lcccc}
\hline & & \multicolumn{3}{c}{ Change in mean JHE in } \\
\cline { 3 - 5 }$r_{\mathrm{G}}$ & $r_{\mathrm{P}}$ & Population & SW morph & LW morph \\
\hline 0.28 & 0.58 & 3.5 & -4.7 & -1.7 \\
0.9 & 0.58 & 11.3 & 3.1 & 6.1 \\
0.9 & 0.95 & 11.3 & -4.2 & 6.5 \\
\hline
\end{tabular}

morphs is, by virtue of the environmental correlation, a function of the phenotypic correlation (eqn 7). The change in JHE activity within morph also depends upon the values of the two heritabilities. For example, in the 13 parameter combinations shown in Table 1 there is always a predicted decrease in JHE activity within the micropterous morph $(\bar{x}=-1.3$, range -0.1 to -2.6$)$, but a predicted increase in the macropterous morph $(\bar{x}=2.6$, range $1.7-3.5)$.

To our knowledge there are two examples of the use of quantitative genetics to make quantitative predictions about the change in trait value in a natural population (Morris, 1971; Grant \& Grant, 1993), but no analysis has predicted the correlated response to selection. Our results use quantitative genetic parameters estimated in the laboratory to predict successfully geographical variation among natural populations. We have used only the standard equation for correlated response to selection, modified to account for a threshold trait. The approach thus has general application wherever it can be hypothesized that selection acts on one trait that is genetically correlated to another. In some cases selection could have acted on both traits, in which case it will be necessary to use the equation incorporating both direct and indirect effects (see chapter 5, Roff, 1997). In addition to predicting changes in population mean values, the quantitative genetic model used here allows us to predict, and hence understand, complex patterns of coevolution such as the counter-intuitive decline of JHE activity within morph, that occurs in spite of an overall increase in JHE activity. This study is thus a clear example of the utility of quantitative genetics as a model of microevolutionary changes in natural populations.

\section{Acknowledgements}

We are grateful for the technical assistance of Martin Cayer. This research was supported by a collaborative grant from NSERC to D.A.R. and D.J.F.

\section{References}

ALEXANDER, R. D. 1968. Life cycles, specialization and related phenomena in crickets. Q. Rev. Biol., 43, 1-41.

BERTHOLD, P. 1995. Microevolution of migratory behaviour illustrated by the blackcap Sylvia atricapilla: 1993 Witherby Lecture. Bird Study, 42, 89-100.

BERTHOLD, P., HELBIG, A. J., MOHR, G. AND QUERNER, U. 1992. Rapid microevolution of migratory behaviour in a wild bird species. Nature, 360, 668-669.

COX, D. R. AND HINKLEY, D. V. 1974. Theoretical Statistics. Chapman \& Hall, London.

Dobson, A. J. 1983. An Introduction to Statistical Modelling. Chapman \& Hall, London.

FAIRBAIRN, D. J. AND YADLOWSKI, D. E. 1997. Coevolution of traits determining migratory tendency: correlated response of a critical enzyme, juvenile hormone esterase, to selection on wing morphology. J. Evol. Biol., 10, 495-513.

FALCONER, D. S. 1989. Introduction to Quantitative Genetics, 3rd edn. Longmans, New York.

Gould, s. J. 1977. Ontogeny and Phylogeny. Belknap Press, Cambridge, MA.

GRANT, B. R. AND GRANT, P. R. 1993. Evolution of Darwin's finches caused by a rare climatic event. Proc. R. Soc. B, 251, 111-117.

HARRISON, R. G. 1985. Barriers to gene exchange between closely related cricket species. II. Life cycle variation and temporal isolation. Evolution, 39, 244-259.

HEWS, D. K., KNAPP, R. AND MOORE, M. C. 1994. Early exposure to androgens affects adult expression of alternative male types in tree lizards. Horm. Behav., 28, 96-115.

KEVAN, D. McE. 1980. The taxonomic status of the Bermuda beach cricket (Orthoptera: Gryllidae). Syst. Ent., 5, 83-95.

LYNCH, M. AND WALSH, B. 1998. Genetics and Analysis of Quantitative Traits. Sinauer Associates, Sunderland, MA.

MATHER, K. AND JINKS, J. L. 1982. Biometrical Genetics, 3rd edn. Chapman \& Hall, London.

MORRIS, R. F. 1971. Observed and simulated changes in genetic quality in natural populations of Hyphantria cunea. Can. Ent., 103, 893-906.

MOUSSEAU, T. A. AND ROFF, D. A. 1987. Natural selection and the heritability of fitness components. Heredity, 59, 181-198.

ROFF, D. A. 1986. The genetic basis of wing dimorphism in the sand cricket, Gryllus firmus and its relevance to the evolution of wing dimorphisms in insects. Heredity, 57, 221-231.

ROFF, D. A. 1989. Exaptation and the evolution of dealation in insects. J. Evol. Biol., 2, 109-123.

ROFF, D. A. 1994. Evidence that the magnitude of the trade-off in a dichotomous trait is frequency dependent. Evolution, $\mathbf{4 8}$, 1650-1656.

ROFF, D. A. 1996. The evolution of genetic correlations: an analysis of patterns. Evolution, 50, 1392-1403.

ROFF, D. A. 1997. Evolutionary Quantitative Genetics. Chapman \& Hall, New York.

ROFF, D. A. AND BRADFORD, M. J. 1998. The evolution of shape in the wing dimorphic cricket, Allonemobius socius. Heredity, 80, 446-455. 
ROFF, D. A., STIRLING, G. AND FAIRBAIRN, D. J. 1997. The evolution of threshold traits: a quantitative genetic analysis of the physiological and life history correlates of wing dimorphism in the sand cricket. Evolution, 51, 1910-1919.

SOUTHwoOD, T. R. E. 1961. A hormonal theory of the mechanism of wing polymorphism in Heteroptera. Proc. $R$. Entomol. Soc. Lond., 36, 63-66.

VEAZEY, J. N., KAY, C. A. R., WALKER, T. J. AND WHITCOMB, W. H. 1976. Seasonal abundance, sex ratio and macroptery of field crickets in northern Florida. Ann. Ent. Soc. Am., 69, 374 380 .

WEIGENSBER, G., I. AND ROFF, D. A. 1996. Natural heritabilities: can they be reliably estimated in the laboratory? Evolution, 50, 2149-2157.

WIGGLESWORTH, V. B. 1961. Insect polymorphism - a tentative synthesis. Symp. Roy. Entomol. Soc. Lond., 1, 103-113.

ZERA, A. J. AND HOLTMEIER, C. L. 1992. In vivo and in vitro degradation of juvenile hormone-III in presumptive long- winged and short-winged Gryllus rubens. J. Insect Physiol., 38, 61-74.

ZERA, A. J. AND TIEBEL, K. C. 1989. Differences in juvenile hormone esterase activity between presumptive macropterous and brachypterous Gryllus rubens: implications for the hormonal control of wing polymorphism. J. Insect Physiol., 35, 7-17.

ZERA, A. J. AND TOBE, S. S. 1990. Juvenile hormone-III biosynthesis in presumptive long-winged and short-winged Gryllus rubens: implications for the endocrine regulation of wing dimorphism. J. Insect Physiol., 36, 271-280.

ZERA, A. J., STRAMBI, C., TIEBEL, K. C., STRAMBI, A. AND RANKIN, M. A. 1989. Juvenile hormone and ecdysteroid titres during critical periods of wing morph determination in Gryllus rubens. J. Insect Physiol., 35, 501-511.

ZERA, A. J., SALL, J. AND GRUDZINSKI, K. 1997. Flight-muscle polymorphism in the cricket Gryllus firmus: muscle characteristics and their influence on the evolution of flightlessness. Physiol. Zool., 70, 519-529. 